\title{
The Shape Identification of Membership Functions by Genetic Algorithm and Fuzzy Control of a Fluid Temperature System
}

\author{
Hee-Hyol LEE \\ Department of Management and System Engineering \\ Fukuoka Institute of Technology \\ 3-30-1 Wajirohigashi, Higashi-ku, Fukuoka 811-02 JAPAN
}

\begin{abstract}
Fuzzy control has been applied to various fields because it is easy to describe experience and knowledge of human directly. This paper deals with a fuzzy control for a fluid temperature system using optimized shapes of membership functions of fuzzy production rules. The shapes of membership functions are optimized through the learning based on the genetic algorithm. The effectiveness of the fuzzy control with the optimized shapes is confirmed by an experiment of a fluid temperature system.
\end{abstract}

\section{Introduction}

On fuzzy inference we can describe ambiguous words into the inference rules of if-then type and then design control systems easily. However it is required of producing the inference rules automatically with the progress of practical use of fuzzy control.

This paper is concerned with the problem of shape identification of membership functions for fuzzy production rules. The shape of the membership functions is optimized through the learning based on the genetic algorithm.

In conformation of the efficiency of the membership functions an experiment of the fuzzy control for a fluid temperature system is carried out.

\section{The Shape Identification of Membership Functions by the Genetic Algorithm}

\subsection{The Procedure of the Genetic Algorithm}

The genetic algorithm, which is effective to the optimal problem, has attracted special interest recently. It follows the phenomenon of natural selection made through the countless generations using crossover and mutation of genes.

The procedure of the genetic algorithm used in this paper is below.

1) As an initial population, 100 individuals are produced, where alleles of a gene of the individuals are selected randomly.

2) Simulation of fuzzy control is carried out.

3) Settling time as a fitness value is calculated.

4) By selection, 30 individuals are erased in the order of the low fitness value.

5) By crossover propagation, 30 individuals are propagated using a uniform cross-over.

6) Mutation is occurred to every individual of probability 10 percent to produce the individual independent of the initial population.

7) Go to 2).

One generation is from 2) to 7), and then the procedure is continued to the last generation.

\subsection{The Shape Identification of Membership} Functions of Fuzzy Production Rules 
The fuzzy production rules are formed of the condition part variables $\mathrm{E}$ and $\Delta \mathrm{E}$, and the operation part variable $\Delta \mathrm{U}$,

rule $i$ : if $E$ is $A_{i 1}$ and $\Delta E$ is $A_{i 2}$ then $\Delta U$ is $B_{i}$ $i=1,2, \cdots, n$

where $\mathrm{E}$ is the error between a reference and a controlled output, $\Delta \mathrm{E}$ is its variation for a sampling time, and $\Delta \mathrm{U}$ is the variation of a control input $\mathrm{U}$. The membership functions of their variables are triangles in this paper.

For the purpose of deducing optimized shape of the membership functions, vertexes of the triangles are moved horizontally. For the parallel displacement, we define an elementary varying quantity $V_{i}$, and give variety of integral multiples of $V_{i}$ to the vertexes. The elementary varying quantity $\mathrm{V}_{\mathrm{i}}$ of the variables $\mathrm{E}, \Delta \mathrm{E}$ and $\Delta \mathrm{U}$ is $1 / 16$ of the base of one triangle as follows,

$$
V_{i}=X_{i} / 48, \quad i=E, \Delta E, \Delta U
$$

where $\mathrm{X}_{\mathrm{i}}$ is the overall width of the condition part variables $\mathrm{E}$ and $\Delta \mathrm{E}$, the operation part variable $\Delta \mathrm{U}$, respectively, and illustrated in Figure 2.1 as an example. There are 19 vertexes with the exception of two end points for the seven fuzzy variables, so that one gene is expressed by 57 alleles. The representation of a gene with the alleles is shown in Figure 2.2.
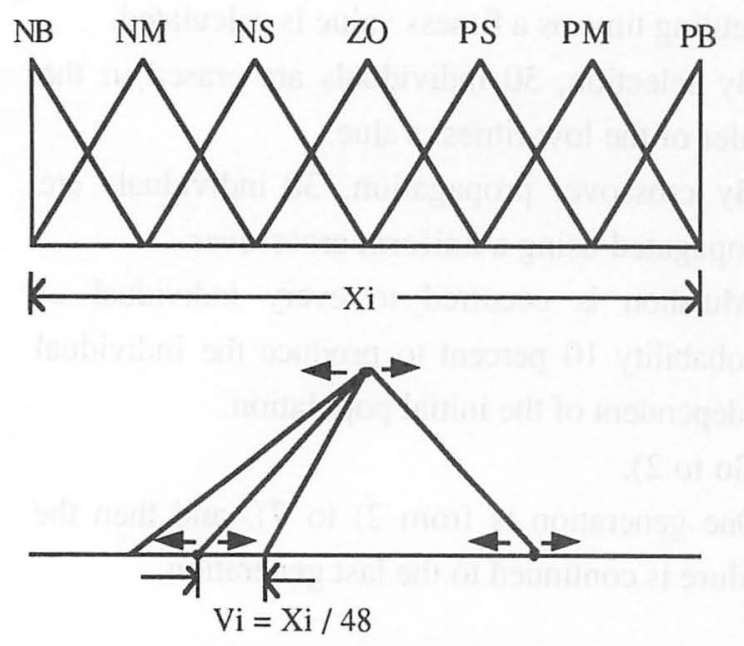

Fig.2.1 Parallel displacement of vertexes by the elementary varying quantity.
On the initial population, one individual with the alleles $\mathrm{a}_{\mathrm{ij}}=\{-2,-1,0,1,2\}$, which is taken randomly, is occurred and the aggregate individuals are 100 , where one individual corresponds to one fuzzy controller.

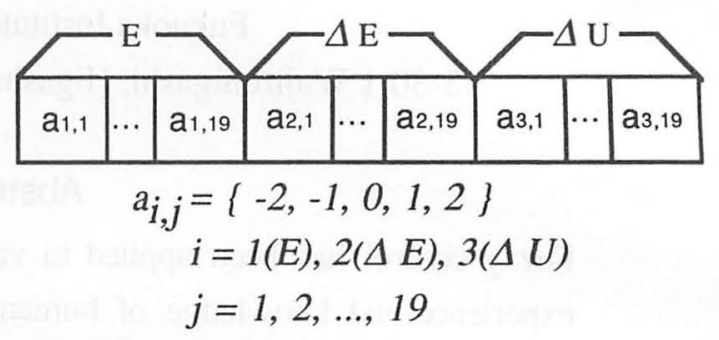

Fig.2.2 Representation of a gene.

Next, simulation of the fuzzy control is carried out for every individual in the initial population, and then the settling time is obtained. The settling time is taken as the fitness value.

On selection, 30 individuals are erased in the order of the low fitness value.

After the selection, the propagation must be carried out because the aggregated number of the individuals in one generation is fixed from the first generation to the last one. On crossover propagation, a uniform cross-over is used based on a mask. Parents A and B are selected first, and if an allele of the mask is 1 then a child inherit the allele from the parent $\mathrm{A}$, while if an allele of the mask is 0 then the child inherit the allele from the parent $B$. The mask is $\{1,1,0,0,0,1,1,1,1,1$, $1,0,0,0,1,1,1,0,0\}$ and 30 individuals as parents $\mathrm{A}$ are selected in the order of the high fitness value and 30 individuals as parents $B$ are selected randomly from the remaining individuals. On the crossover propagation, 30 children are propagated and the parents $\mathrm{A}$ and $\mathrm{B}$ remain in the next generation.

Mutation is occurred to every individual of probability 10 percent to produce the individual independent of the initial population.

These procedures are continued from the first generation to the last generation. 


\subsection{The Estimation of Mathematical Model of a Fluid Temperature System}

Before the simulation of the fuzzy control to calculate the fitness value of the individual a mathematical model is necessary. At the estimation of the mathematical model the input signals to the control system, which is a fluid temperature experimental plant, are warm water $70\left[{ }^{\circ} \mathrm{C}\right]$ driven by $\{0,1\}$ due to normal random numbers, and the output signals are the measured temperature of the control system, where the sampling time is 8 [sec]. There are trends obviously in the input and output signals, so the trends were removed using the third order equation. Figure 2.3 is the input and output signals to estimate the mathematical model of the control system.
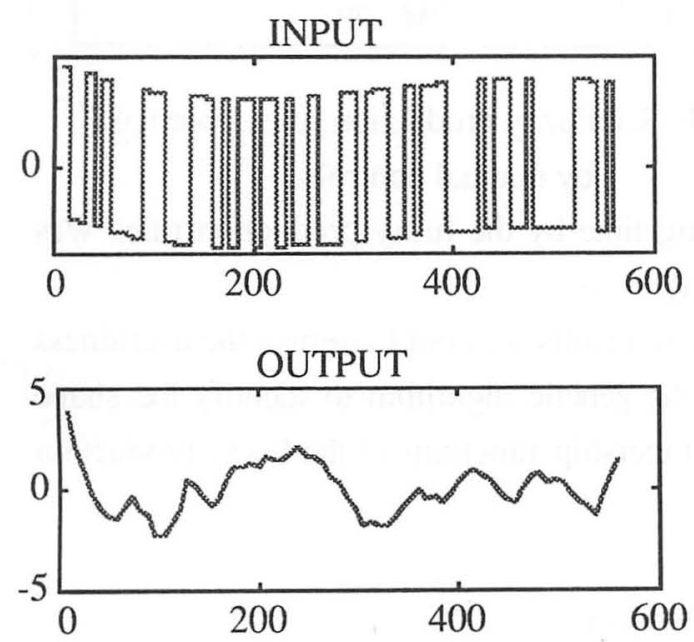

Fig.2.3 Input and output signals for estimation of the mathematical model.

Using LMS and AIC the estimated mathematical model is

$G(z)=z^{-2} \frac{0.14+0.57 z^{-1}+0.28 z^{-2}-0.01 z^{-3}-0.16 z^{-4}}{1-0.97 z^{-1}-0.22 z^{-2}+0.17 z^{-3}+0.10 z^{-4}}$

and the third order equation of the trend of the output signals is

$y(k T)=2.3 \times 10^{-7}(k T)^{3}-3.9 \times 10^{-4}(k T)^{2}+2.0 \times 10^{-1}(k T)+11.92$
The step response of the estimated model added the trend and the step response of the fluid temperature experimental plant are shown in Figure 2.4.

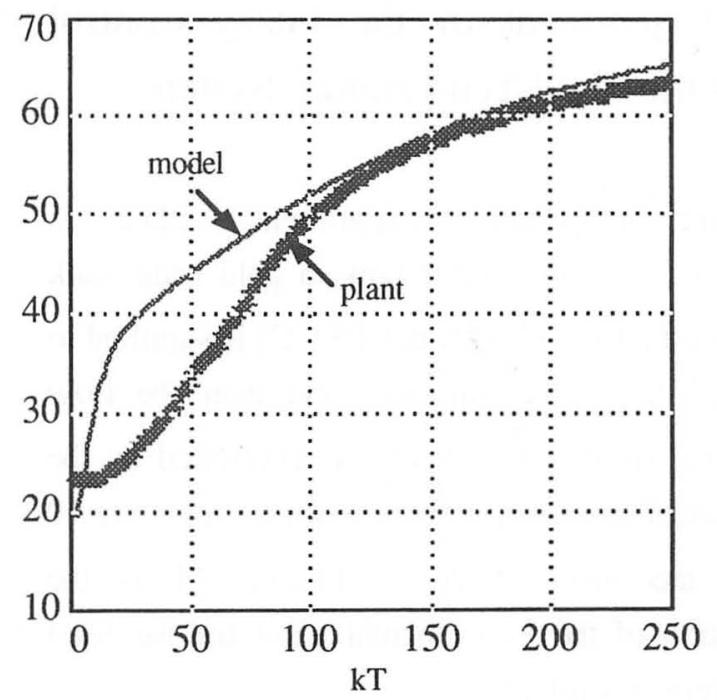

Fig.2.4 Step responses of the model and plant.

\subsection{The Membership Function obtained by the Genetic Algorithm}

As the result of the procedure of the genetic algorithm by 2,000 generations and the estimated mathematical model mentioned above, the membership functions of the condition part variables $\mathrm{E}$ and $\Delta \mathrm{E}$, and the operation part variable $\Delta \mathrm{U}$ are illustrated in Figure 2.5.

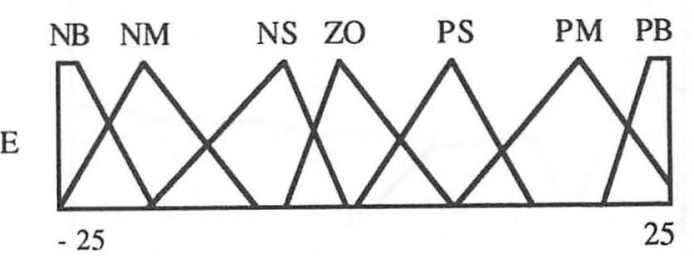

$\Delta \mathrm{E}$

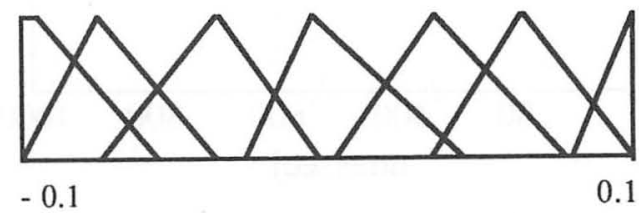

$\Delta \mathrm{U}$

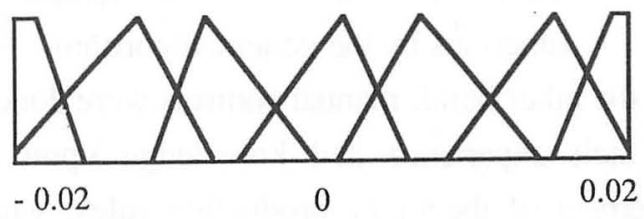


Fig.2.5 The membership functions obtained by the genetic algorithm.

\section{The Experiment of the Fuzzy Control} using the Fluid Temperature System

The fluid temperature experimental plant is composed of a warm water tank, a cold water tank and a mixed tank. Cold water $15\left[{ }^{\circ} \mathrm{C}\right]$ is supplied to the mixed tank at a uniform, and then the flow quantity of warm water $70\left[{ }^{\circ} \mathrm{C}\right]$ is controlled by the fuzzy controller to keep the reference temperature 40 $\left[{ }^{\circ} \mathrm{C}\right]$ of the mixed tank. Figure 3.1 is the construction of the experimental plant for the fluid temperature control system.

The controlled output of the fuzzy control based on the membership functions obtained by the genetic algorithm is shown in Figure 3.2, and the settling time within \pm 5 percent of the reference value is 3.72 [min].

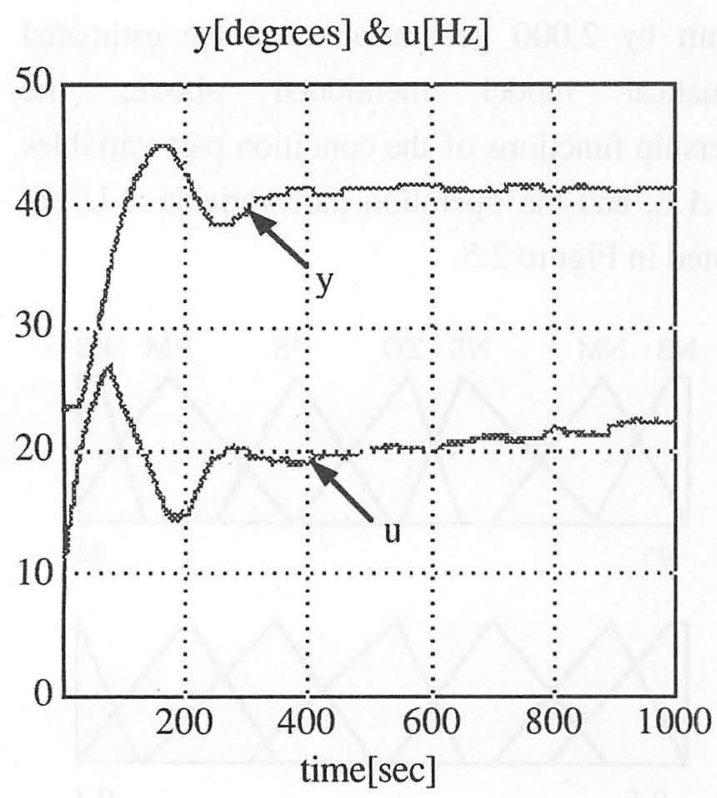

Fig.3.2 The fuzzy control with the membership functions by the genetic algorithm.

On the other hand, manual controls were done to reflect their experience and knowledge upon the improvement of the fuzzy production rules, where the vertexes of the triangles of the membership function are not moved. Table 3.1 is the fuzzy production rules improved by the experiment by the dozens. Some rules are added to get a rise of the response and a decrease of the overshoot as follows:

$$
\begin{aligned}
& \text { if } \mathrm{E}=\mathrm{PB} \text { and } \Delta \mathrm{E}=\mathrm{NS} \text { then } \Delta \mathrm{U}=P M \\
& \text { if } \mathrm{E}=\mathrm{PM} \text { and } \Delta \mathrm{E}=\mathrm{NM} \text { then } \Delta \mathrm{U}=N S \\
& \text { if } \mathrm{E}=\mathrm{PS} \text { and } \Delta \mathrm{E}=\mathrm{NS} \text { then } \Delta \mathrm{U}=N S \\
& \text { if } \mathrm{E}=\mathrm{NS} \text { and } \Delta \mathrm{E}=\mathrm{PS} \text { then } \Delta \mathrm{U}=P S \\
& \text { if } \mathrm{E}=\mathrm{PS} \text { and } \Delta \mathrm{E}=\mathrm{PS} \text { then } \Delta \mathrm{U}=P S
\end{aligned}
$$

\begin{tabular}{|c|c|c|c|c|c|c|c|c|}
\hline & \multicolumn{7}{|c|}{$\Delta \mathrm{E}$} \\
\hline & & NB & NM & NS & $\mathrm{ZO}$ & PS & $\mathrm{PM}$ & $\mathrm{PB}$ \\
\hline & NB & & & & NB & & & \\
\hline & NM & & & & $\mathrm{NM}$ & & & \\
\hline & NS & & & & NS & $P S$ & & \\
\hline \multirow[t]{4}{*}{ E } & $\mathrm{ZO}$ & $\mathrm{NB}$ & NM & NS & $\mathrm{ZO}$ & PS & PM & PB \\
\hline & PS & & & $N S$ & PS & $P S$ & & \\
\hline & PM & & $N S$ & & PM & & & \\
\hline & PB & & & $P M$ & PB & & & \\
\hline
\end{tabular}

Table 3.1 Fuzzy production rules improved by manual controls.

The settling time by the fuzzy production rules was 3.58 [min].

As these results we could confirm the usefulness of using the genetic algorithm to identify the shape of the membership functions of the fuzzy production rules.

\section{Conclusion}

In this paper, we proposed a shape identification of fuzzy production rules using the genetic algorithm. An experiment of the fuzzy control for a fluid temperature system showed the effectiveness of the membership functions obtained by the genetic algorithm.

\section{References}

[1] E.Mamdani: Advances in the linguistic synthesis of fuzzy controller, Int.J.Man-Machine Studies, 
Vol.8, No.6, pp.669-678, 1976

[2] Holland, J.H.: Adaptation in natural and artificial system, The University of Michigan press, 1975

[3] Y.Yonezawa: Genetic algorithm, Morikita Shuppan, 1993
[4] S.Sagara, K.Akizuki, T.Nakamizo, T.Katayama: System Identification, SICE, 1981

[5] M.Sugeno: Fuzzy Control, Nikkan Kougyou Sinbunsha, 1988

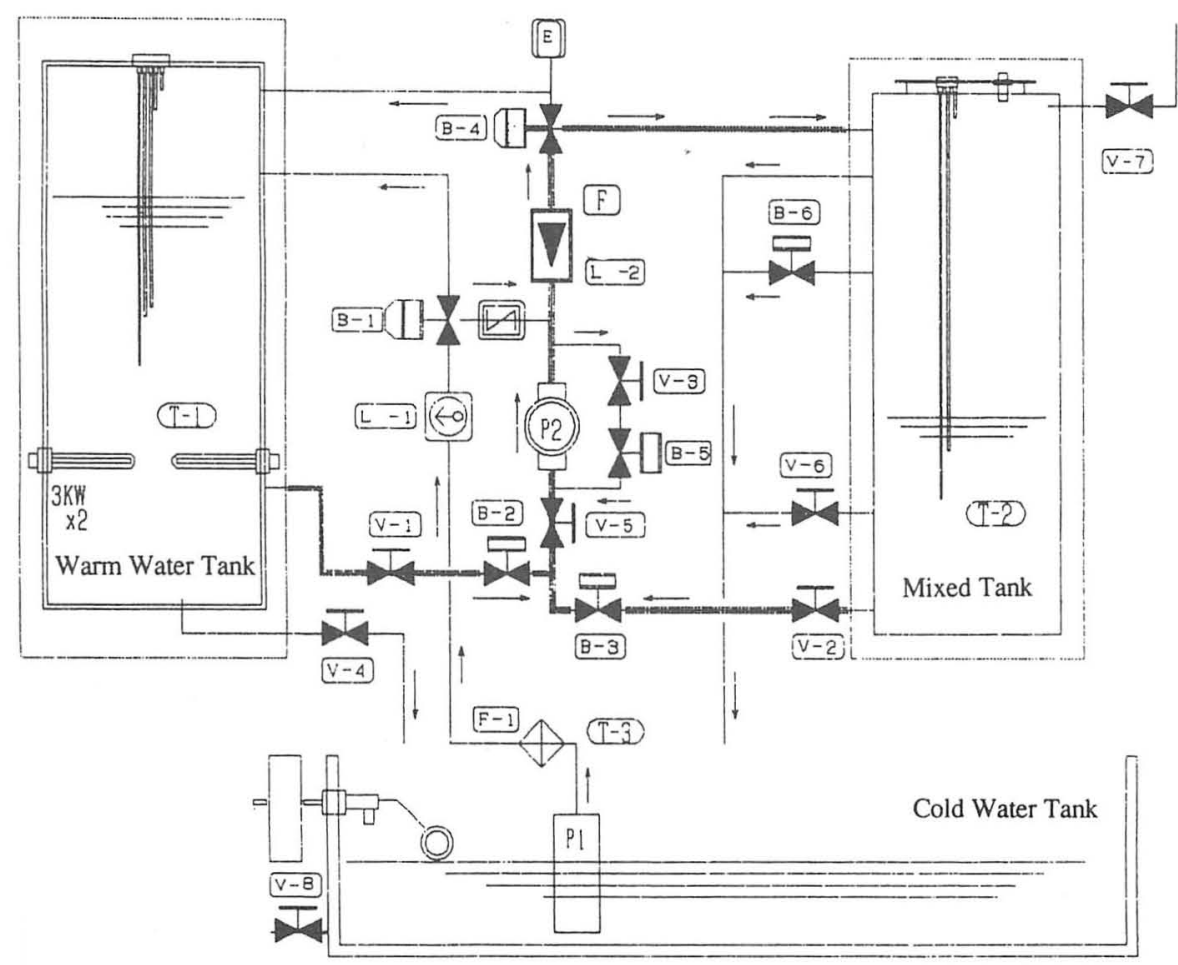

Fig.3.1 Construction of the experimental plant for the fluid temperature control system. 
